\title{
Using Automated Suture Fastening System For Securing Aortic Cannulation Sites In Minimally-Invasive Mitral Valve Surgery
}

\author{
Abdullah Nasif ${ }^{1}$ and Saqib Masroor ${ }^{1}$ \\ ${ }^{1}$ The University of Toledo Department of Surgery
}

February 11, 2022

\begin{abstract}
Cor-Knot device is an automated suture fastening system which was introduced to minimize the operating time during cardiac surgery. It is currently being used for intracardiac suturing applications such as implanting valves or rings during aortic and mitral valve surgery primarily for shortening the procedure time. It also has higher holding pressures and more consistent holding pressures than manual knots. We describe here the use of Cor-Knot device in extracardiac suturing to secure aortic root vent as well as aortic cannulation during minimally invasive cardiac surgery. A 60-year-old male with mitral valve endocarditis underwent minimally invasive mitral valve replacement via right mini thoracotomy. Direct ascending aortic cannulation was performed through a separate port. Cor-Knots were successfully used to secure the purse-string sutures after decannulation at the end of the case.
\end{abstract}

\section{Introduction:}

Cor-Knot (LSI Solutions, Victor, NY) is an automated suture fastening system introduced in 2008 to eliminate manual knot-tying for valvular prosthesis implantation. It also has higher and more consistent holding pressures than manual knots, a quality that would make it a better suturing system for high pressure sites such as aorta. However, aortic root vents and aortic cannulas are typically secured with sutures that are tied with 6 or more knots. During minimally invasive surgery, these knots are tied outside the chest and then slid down on to the aorta. There are instances when the aortic cannulation sites require additional sutures to control residual leaks from an 'air-knot'. There are also instances of re-exploration for bleeding from these areas. Based on the reports of better holding pressure, we have routinely used Cor-knots for securing aortic root vent/antegrade cardioplegia catheter during minimally invasive procedures for the past 4 years, without any complications related to the suture or the fastener. Here we describe the first reported case of using Cor-Knot for securing large-bore cannula for direct aortic cannulation in minimally-invasive mitral valve surgery.

\section{Methods and methods:}

Patient chart was reviewed for complete history and details of his operative details and postoperative course. The Institutional Review Board (IRB) approval was not obtained, since single case report with deidentified data does not require IRB approval. The patient provided informed written consent for the publication of the study data.

\section{Results:}

A 60-year-old male with enterococcus faecalis bacteremia, mitral valve endocarditis, and very large mobile mass on the mitral valve underwent minimally invasive mitral valve replacement with $29 \mathrm{~mm}$ Magna Ease pericardial valve via right mini-thoracotomy (RMT). Due to severe atherosclerosis in the iliofemoral vessels, direct aortic access was needed through the chest. Two purse-string stitches were placed in the standard 
fashion through the RMT incision and then pulled out of the chest through a 12-mm port placed in the second intercostal space. Using the Seldinger technique, a guidewire was introduced into the distal ascending aorta within the pursestring via a needle placed through the RMT. The wire was also pulled out through the port. After serial dilation over the wire, a 20-French cannula was advanced into the aortic arch and secured to the purse-string sutures. TEE confirmed the position of the wire and cannula in the aortic arch. Cardiopulmonary bypass was initiated and antegrade cardioplegia catheter was placed in the ascending aorta. Surgery proceeded in the usual fashion. After excising the infected valve, a $29 \mathrm{~mm}$ pericardial valve was implanted using Cor-Knot device. After de-airing, aortic root vent was removed and the site was secured with a Cor-Knot (Figure 1A and 1B). The patient was weaned off cardiopulmonary bypass. Aortic cannula was removed and the aortotomy purse-string sutures were secured separately with two additional Cor-Knots through the port under vision (Figure 2). There was no bleeding from the cannulation or the root vent site. Patient was discharged from hospital in 15 days after undergoing femoral-to-femoral bypass and iliofemoral endarterectomy for lower extremity ischemia on post-op day 8.

\section{Discussion:}

Core-Knot has become popular in valvular surgery since its introduction in 2009. While reducing cardiopulmonary bypass time is its prime benefit, it has also been found to have better holding pressure than manual knot tying. ${ }^{1}$ Lee et al. evaluated the strength, consistency, and speed of prosthetic attachment sutures secured with Cor-Knot and compared with those of manual knots. ${ }^{1}$ They reported that suture holding pressures for the Cor-Knot fasteners were significantly greater (median, 1008.9 vs $415.8 \mathrm{~mm} \mathrm{Hg}, \mathrm{P}<0.001$ ). Also, there was less variation in attachment pressures with significant time savings occurred with the use of the Cor-Knot compared with manual tying (12.4 vs 71.1 seconds per knot, $\mathrm{P}=0.001$ ). They concluded that mitral annuloplasty ring sutures secured with the Cor-Knot device were stronger, more consistent, and faster than with manually tied knots. ${ }^{1}$

One of the first reported studies on Cor-Knot system was conducted between 2008-2010. Rodriguez et al. evaluated the clinical outcomes of minimally invasive mitral valve surgery with prosthetic attachment sutures secured by Cor-Knot system. ${ }^{2}$ No patient had evidence of thrombus formation, hemolysis, or perivalvular leaks. $^{2}$ One instance of mitral ring dehiscence was noted, but the dehiscence was due to a tear in the mitral annulus and not associated with the titanium fasteners, which all remained intact. ${ }^{2}$ They concluded that at a minimum of one-year follow-up, Cor-Knot system is safe and effective for securing prosthetic attachment suture. $^{2}$

Sazzad et al. quantified the incidence of valvular complications related to Cor-Knot device. ${ }^{3}$ They reported trivial and/or mild paravalvular leak (PVL) in immediate post-operative echocardiography and no reported cases of valvular thrombosis, leaflet perforation, device dislodgement or embolization, moderate and/or severe PVL during hospitalization and follow-up echocardiography within 1 year. $^{3}$

Lee et al. analyzed prosthetic fixation pressures in prosthetic aortic valves, using Digital Pressure Mapping with $2 \mathrm{D}$ and $3 \mathrm{D}$ pressure maps generated for all valves. None of the 288 Cor-Knot cases had any decrease in intra-suture pressure, while 12 out of 144 manual-tying cases that had pressure drop to less than $80 \mathrm{mmHg}$ which is considered a risk for paravalvular leaks (PVL) $(\mathrm{P}<0.001) .{ }^{4}$ Therefore, it would be reasonable to use this device at high pressure sites such as the aorta.

\section{Conclusion:}

Cor-Knot device is currently being used for intracardiac applications such as implanting pericardial valve in minimally-invasive mitral valve replacement. We have now utilized the Cor-Knot automated suture fastening system to close small caliber sites in aorta, such as root vents, instead of traditional suturing technique for over 4 years without any suture site complications. In this report we have demonstrated the safety of using this suture fastener system on large bore aortic cannulation sites also. One of the benefits of using this device is that it allows direct visualization of the site as the sutures are being tightened. This should be considered for direct aortic cannulation not only in minimally invasive but also open surgery where this provides controlled deployment under direct vision, rather than manual suturing with "feeling" the tightness 
of the knot by the surgeon's hands.

\section{Author contribution statement:}

Abdullah Nasif: Data curation, Writing-original draft, Writing-review \& editing, Visualization. Saqib Masroor: Conceptualization, Investigation, Supervision, Validation, Writing-review \& editing.

\section{Figure/Video legend:}

Figure 1. A) Aortic root vent site secured with a Cor-Knot device. B) Chest CT shows the clip on aortic vent site.

Figure 2. Chest CT shows the two clips on aortic cannulation site with 615 Hounsfield unit (HU) indicating high density material (Metal).

Video 1. Cor-Knot deployment at the aortic vent site.

\section{References:}

1. Lee CY, Sauer JS, Gorea HR, Martellaro AJ, Knight PA. Comparison of strength, consistency, and speed of COR-KNOT versus manually hand-tied knots in an ex vivo minimally invasive model. Innovations (Phila). 2014 Mar-Apr;9(2):111-6; discussion 116. PMID: 24755535.

2. Rodriguez R, Sutter FP, Samuels LE, Ice D, Morrissey S, Holzerman KM, Goldman S. Clinical Outcomes of Patients More Than One Year after Minimally Invasive Mitral Valve Repair and Replacement Surgery with Sutures Secured using Automated Titanium Fasteners. ISMICS. 2013 June 12-15.

3. Sazzad F, Xian OZ, Ler A, Guohao C, Swee KG, Kofidis T. Incidence of valvular regurgitation and leaflet perforation by using automated titanium fasteners (CORKNOTR) in heart valve repair or replacement: less usual than reported. J Cardiothorac Surg. 2021 Jun 7;16(1):163. PMID: 34099017; PMCID: PMC8186203.

4. Lee CY, Wong JK, Ross RE, Liu DC, Khabbaz KR, Martellaro AJ, Gorea HR, Sauer JS, Knight PA. Prosthetic Aortic Valve Fixation Study: 48 Replacement Valves Analyzed Using Digital Pressure Mapping. Innovations (Phila). 2016 Sep/Oct;11(5):327-336. PMID: 27561176.

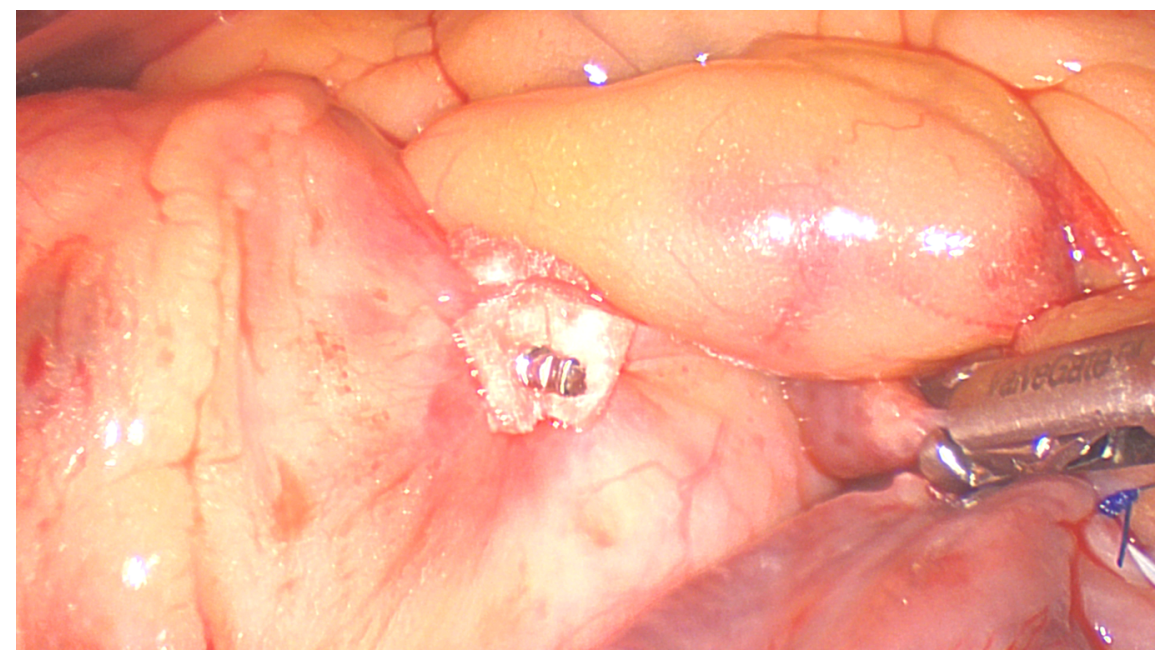



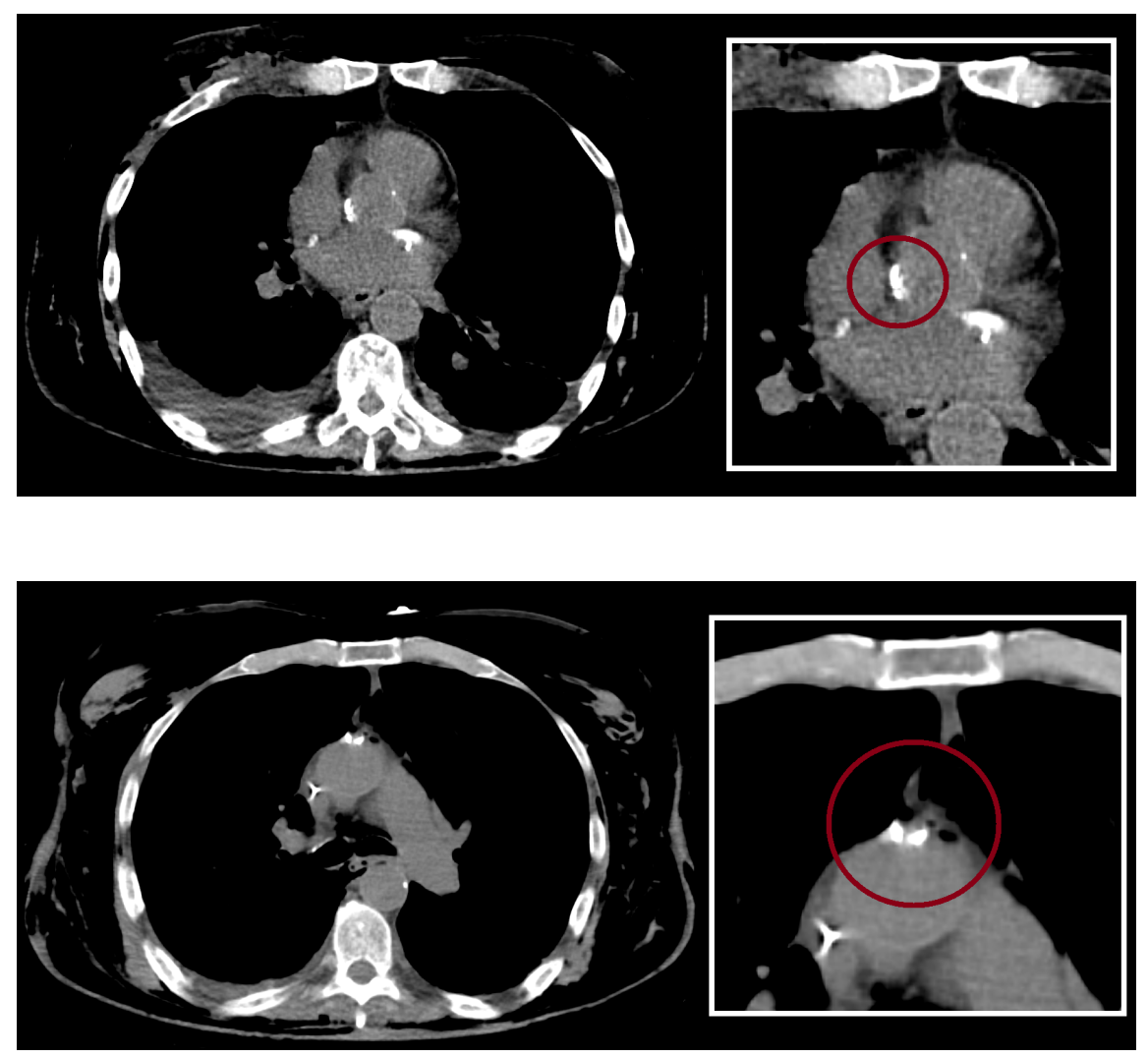

Hosted file

CorKnot - Video 1.mp4 available at https://authorea.com/users/415675/articles/556110-usingautomated-suture-fastening-system-for-securing-aortic-cannulation-sites-in-minimallyinvasive-mitral-valve-surgery 